Archives

$44 \mid 2009$

Varia

\title{
Les pratiques de la lecture chez Michel de Certeau
}

La matérialité de l'œuvre

Andrés G. Freijomil

\section{(2) OpenEdition}

Journals

Édition électronique

URL : http://journals.openedition.org/ccrh/3533

DOI : $10.4000 /$ ccrh.3533

ISSN : $1760-7906$

Éditeur

Centre de recherches historiques - EHESS

Édition imprimée

Date de publication : 25 janvier 2009

Pagination : 109-134

ISSN : 0990-9141

\section{Référence électronique}

Andrés G. Freijomil, «Les pratiques de la lecture chez Michel de Certeau », Les Cahiers du Centre de Recherches Historiques [En ligne], 44 | 2009, mis en ligne le 29 novembre 2011, consulté le 19 avril 2019. URL : http://journals.openedition.org/ccrh/3533; DOI : 10.4000/ccrh.3533

Ce document a été généré automatiquement le 19 avril 2019.

Article L.111-1 du Code de la propriété intellectuelle. 


\section{Les pratiques de la lecture chez Michel de Certeau}

La matérialité de l'œuvre ${ }^{1}$

Andrés G. Freijomil

Ainsi du lecteur : son lieu n'est pas ici ou là, l'un ou

l'autre, mais ni l'un ni l'autre, à la fois dedans et dehors, perdant l'un et l'autre en les mêlant, associant des textes gisants dont il est l'éveilleur et

l'hôte, mais jamais le propriétaire. Michel de Certeau

1 Dans le cadre d'une recherche consacrée à l'œuvre de Michel de Certeau, rien ne semble plus adéquat - et d'une certaine manière plus juste - que de commencer par sa « prise de parole ». Néanmoins, l'épigraphe reproduite ci-dessus consigne non seulement l'un des points clés de sa "poétique du braconnage », c'est-à-dire, celui de la « déterritorialisation » d'un lecteur qui ne cesse de voyager, mais aussi l'impression d'un inquiétant trompe-l'œil : le replacement constant que tout chercheur est obligé de suivre au moment de conduire une étude de son œuvre. Si l'on ajoute à cela l'intention d'enquêter sur ses pratiques de la lecture, le dessein se présente encore plus téméraire. C'est qu'en effet, soit par la bordure "traversière" de sa conception du travail intellectuel (pour reprendre l'épithète utilisée par Louis Marin) ${ }^{2}$, soit par la zone que nous abordons de son œuvre, on est face à un « sujet » et à un « objet » également fuyants. La première exigence qui s'impose est donc de multiplier les perspectives d'analyse et $d$ ' «attraper » ses « arts de braconner » présents partout en examinant d'emblée la nature de son principal legs : son œuvre.

\section{Une poétique du « réemploi »}

2 Au premier abord, il s'agit d'un examen nécessaire en raison d'une des caractéristiques matérielles de l'œuvre certalienne : celle-ci se veut aussi fragmentaire et disséminée que les espaces du savoir que de Certeau a franchis. C'est dire qu'il n'a pas écrit uniquement 
des ouvrages, mais notamment des articles ou des essais pour un grand nombre de revues spécialisées ou adressées à un public plus large. C'est ce premier laboratoire qui donne lieu à la matérialité de presque tous ses livres à partir du « réemploi » de textes déjà publiés ou d'autres encore inédits. De cette façon, et comme s'il s'agissait des pièces d'un puzzle, un article paru dans une revue pouvait reparaître ensuite corrigé ou fragmenté dans une autre ou devenir le chapitre d'un livre, de telle sorte que tous les grands ouvrages publiés sous son seul nom (sauf La possession de Loudun) ne seraient que des « recueils» soigneusement pensés depuis longtemps pour une communauté de lecteurs différente de celle d'origine. À cet égard, le travail de réécriture que Michel Lafon a observé dans la littérature de Jorge Luis Borges ${ }^{3}$ pourrait bien se trouver sous forme de « réemploi » chez un « disciple » secret (mais rebelle à la fois) comme de Certeau qui, d'ailleurs, fut un grand et précoce lecteur de l'œuvre borgésienne. En tout cas, et bien qu'il soit naturel de perdre de vue cet exercice de "conversion " textuelle et de lire ses livres comme s'il s'agissait du résultat linéaire d'un processus continu et homogène d'écriture, il est nécessaire de considérer cette pratique au moment de pénétrer dans ses ouvrages étant donné qu'il existe une vraie déclaration de principes dans cette matérialité par rapport, notamment, à la présence du lecteur. Comme Irène Langlet l'a souligné à propos du « recueil »,

le lecteur a toujours le dernier mot, et les circuits qu'il peut emprunter dans le recueil renseignent sur des effets de structuration appuyés sur des procédés, des agencements, des dispositifs ${ }^{4}$.

Chacun des ouvrages de Michel de Certeau pourrait fonctionner comme une "économie de lecture ${ }^{5}$ dont l'assemblage n'a pas répondu uniquement à une occasion éditoriale, mais aussi à la conception que lui-même avait du lien entre la figure du « livre » et celle de l'« auteur », entre l'« écriture » et la « lecture », et enfin, tel que l'a remarqué Jacques Le Brun, de la problématique du « texte » dès le début de son parcours intellectuel ${ }^{6}$. C'est pourquoi il faut insister sur l'une des hypothèses qui fonde notre recherche : il serait impossible d'avoir une perception achevée (ou prétendument achevée) des idées certaliennes à travers une lecture linéaire de ses livres publiés étant donné que les enjeux de leur matérialité constituent une partie indissociable d'une gnoséologie où la connaissance n'est pas une simple entité abstraite, mais le produit itinérant d'une expérience matérielle et concrète de lecture et d'écriture.

Dans ce contexte, les attributs des deux dispositifs explicitement formulés par Michel de Certeau sur la lecture (d'une part, le « braconnage » chez « l'homme sans qualités » du xx siècle ${ }^{7}$, de l'autre, la pratique « absolue » chez les mystiques des XVI et XVII siècles) ${ }^{8}$, ne furent pas étrangers à une telle mobilité. C'est dire que de Certeau a fait de la «lecture » une activité praticable, mais également «objectivable». C'est sa capacité singulière à construire des « fictions théoriques » à partir d'une expérience « vécue » ou « lue » qui l'a mené à proposer, à la fin des années soixante-dix, cette " esquisse » à deux fronts de la lecture. Sur ce point, il faut remarquer qu'à l'origine de sa recherche se trouvait non seulement son attirance pour les enjeux de l'altérité (le lecteur deviendra, bien entendu, un « autre » partiellement soumis par « l'idéologie du livre»), mais aussi la lecture de soimême, un exercice dont la pratique deviendra la synthèse ou le manifeste d'un processus qui a commencé par la protohistoire de ses premières lectures et qui est repérable, pour nous, dès ses premiers textes publiés. Enfin, il ne faut pas oublier que nous sommes face àun lecteur qui oriente l'essentiel de ses compétences vers la production d'un texte écrit. 
Comme l'a souligné Michel Foucault à propos de la meditatio et de l'effet que l'on attend de la lecture :

non pas d'avoir compris ce que voulait dire un auteur, mais la constitution pour soi d'un équipement de propositions vraies, qui soit effectivement à soi [...] si la lecture est conçue ainsi comme exercice, expérience, s'il n'y a de lecture que pour méditer, cette lecture soit immédiatement liée à l'écriture [...]. La lecture se prolonge, se renforce, se réactive par l'écriture, écriture qui est elle aussi un exercice, elle aussi un élément de méditation ${ }^{9}$.

5 À ce sujet, il s'agit d'un processus qui nous oblige à reconfigurer les conditions de sa pratique à partir de son activité d'écrivain. En fin de compte, le fait d'étudier de Certeau en tant que lecteur à partir de ce que révèle sa propre œuvre, signifiera prêter attention aux diverses stratégies poétiques à travers lesquelles il a incorporé chacune de ses lectures dans l'écrit, ainsi qu'à une histoire expressive où son écriture deviendra le principal témoignage - bien que jamais la garantie - de sa représentation culturelle et sociale du monde ${ }^{10}$.

6 Pour atteindre cet objectif, il a été nécessaire de lire chacun de ses travaux dispersés dans son lieu de publication d'origine, et non seulement ses livres publiés. Cela revient à se rapprocher au premier abord de ses articles de revue, de ses comptes rendus, de ses collaborations dans des travaux collectifs pour guetter par la suite ses livres à partir de ses premières éditions. À ce sujet, l'un des principaux outils de travail est l'indispensable « Bibliographie complète » que l'historienne Luce Giard a établie en 1988 pour le dossier paru en deux numéros de la revue Recherches de Science Religieuse ${ }^{11}$, puis publié en un ouvrage, Le voyage mystique. Michel de Certeau ${ }^{12}$. Malgré les courtes possibilités de complétude de toute bibliographie (et que Luce Giard assume comme telles) ${ }^{13}$, sa découverte n'a pas concerné seulement le recensement des textes connus, méconnus ou inconnus de Michel de Certeau, mais son étonnant résultat: l'inépuisable travail de « réemploi » qu'il a pratiqué avec une bonne partie de son corpus textuel. Il s'agit là, en fait, du premier grand moment des études certa-liennes, lequel marque non seulement la route d'un ample projet de mise en perspective de sa figure intellectuelle animée par Luce Giard elle-même et un grand nombre d'historiens ${ }^{14}$, mais aussi l'avènement d'une discrète « révolution », inattendue par rapport à la matérialité de ses écrits. À cet égard, l'usage d'un terme si fondamental comme « réemploi » ${ }^{15}$ (et non celui de "réécriture ») n'est pas hasardeux si l'on considère le souci dont avait fait preuve Michel de Certeau face au possible "emprisonnement " qu'un système scripturaire pourrait lui imposer. Chez lui, la pratique de l'écriture est en fait « l'activité concrète qui consiste sur un espace propre, la page, à construire un texte qui a pouvoir sur l'extériorité dont il a d'abord été isolé ${ }^{16}$. C'est ainsi qu'au delà de la surface de la page blanche où le sujet se donne «le champ d'un faire propre ", et des opérations articulées qui donnent lieu au texte (toujours mobile et en marche), il existe un troisième élément qui fait de cette construction une vraie « formalité » : le changement de la « réalité». Autrement dit, si

l'île de la page est un lieu de transit où s'opère une inversion industrielle [en même temps que], l'entreprise scripturaire transforme ou conserve au-dedans ce qu'elle reçoit de son dehors et crée à l'intérieur les instruments d'une appropriation de l'espace extérieur ${ }^{17}$,

7 il serait possible d'envisager chacun de ses « recueils » comme le produit d'une « poétique de l'archipel ${ }^{18}$.

8 Ainsi, tandis que le travail de réemploi essaye de construire une unité symbolique pour l'ensemble en réduisant les degrés d'autonomie que chaque écrit avait dans le passé (à 
l'aide notamment des dispositifs du paratexte) ${ }^{19}$, c'est l'origine matérielle de chacun d'entre eux qui fera de la "cohabitation» un espace pluriel dont le seul et le grand bâtisseur ne sera que son lecteur. Tel sera donc le destin de tout «braconnier» de l'œuvre certalienne : de naviguer avec l'esprit protéen d'un Ulysse homérique, mais en pérégrinant avec la sagacité quotidienne et éphémère propre à un Leopold Bloom ${ }^{20}$.

De cette façon et en tant que philosophème essentiel de sa pensée, le titre de son deuxième " recueil », L'Étranger ou l'union dans la différence révèlera, précisément, l'esprit de cette expérience matérielle de lecture. Dans l'avant-propos à la première édition de l'ouvrage, de Certeau soulignera :

L'analyse consacrée ici à des recherches communes résulte de multiples rencontres

- trop voilées peut-être, une fois réunies en un seul texte. Elle tente de préciser un mouvement de la foi chrétienne. Il serait sans doute plus exact de dire: une structure de l'expérience chrétienne. Mais ce mot de «structure » est trop lourd pour un aussi petit volume, trop abstrait aussi pour l'interrogation personnelle, nouée et dénouée au cours de bien des échanges, qui fait l'objet de ces pages. Un livre est une place publique, de village ou de grande ville, peu importe. On vient et on va. On y passe. On peut y causer. Qu'espérer, sinon que le lecteur, étranger proche, le traverse comme un lieu de communication ${ }^{21}$ ?

Il donne lieu à un dialogue inédit parmi les textes que de Certeau construit à partir d'un dehors conjectural à la seule fin que le lecteur l'exécute. Il en va de même pour l'avant propos de L'absent de l'histoire en 1973 - un autre « recueil» dont les essais remettent, dans ce cas, à lui-même en tant que lecteur -

Le discours d'un autre a rendu possible chacun d'eux. Ils ne constituent pas une œuvre propre, ni la description d'un itinéraire. Ils se placent sur les accotements de mes travaux personnels et au plus près d'études importantes dont je ne suis que le lecteur. Ils ont poussé dans cet entre-deux qui privilégie l'échange des questions plutôt que la vérification de réponses ${ }^{22}$.

11 Ainsi, du flâneur au braconnier, Michel de Certeau fabriquera des « lecteurs » autant que des « croyants » puisque ses livres auront un contenu, et aussi en particulier des formes ordonnées à la recomposition d'un assemblage symbolique que les lecteurs devront découvrir. Il annonce une route, mais il se situe bien éloigné de celle-ci : d'une certaine manière, il s'agit de livres au pluriel dont l'« autorité » reste suspendue jusqu'au moment où le lecteur s'approprie celle-ci, si nous l'entendons comme lui-même l'a définie, c'est-àdire,

tout ce qui fait (ou prétend faire) autorité - représentations ou personnes - et se réfère donc, d'une manière ou d'une autre, à ce qui est « reçu » comme « croyable » 23.

12 C'est pour cela qu'il n'y a pas de "centre ", mais une "voix » qui compose et essaie d'établir le discernement à la «périphérie » de l'ouvrage. De sorte que chacun des essais réunis doit être pris dans sa particularité pour ainsi rendre son sens pensable, lequel, en vérité, ne sera pas non plus unique. À vrai dire, Michel de Certeau donnera souvent l'impression de soumettre le lecteur à une pratique confessionnelle, là où il raconte de petits morceaux de réalité dont la croyance reste suspendue à la manière d'une meraviglia tésaurienne ${ }^{24}$.

13 Quoi qu'il en soit, en ce qui concerne les besoins de notre recherche, il ne faudra pas oublier que le "réemploi » d'un écrit ne permet d'identifier que partiellement le "sens » des textes : à l'intérieur de chaque « récit » se cache la trace de son propre piège ${ }^{25}$, car il existe souterrainement des textes disparus de l'horizon discursif qui font partie tantôt de 
l'idéologie conformée par l'écrit et à laquelle il ne faut plus se référer, tantôt de ce que, directement, il convient de ne pas citer. Cette dialectique dépendra en même temps de la scène que chaque contexte intellectuel trame et qui régit à l'habitude les publications et les intérêts éditoriaux. Comme l'a remarqué Louis Marin, cet autre grand façonneur de « recueils",

un recueillement de fragments qui n'ont de sens que les uns par les autres, que par leur écarts, mais qui, parce qu'ils sont fragments en recueil peuvent être lus, malgré cet avant propos, dans n'importe quel sens. Et en fin de compte, c'est peut-être là leur avantage : lus dans n'importe quel sens, ils auront une chance - celle que nous ne leur avons pas donnée, en les écrivant un à un - de produire d'autres sens ${ }^{26}$.

À cet égard, la dissection des « rites de passage » de l'article (ou de l'essai) au chapitre se montre utile pour dévoiler la représentation que de Certeau avait de lui-même en tant que braconnier de ses propres textes: une modalité qui, dans son cas, est particulièrement valable puisque, à la manière d'un Pierre Ménard borgésien, il a pratiqué la lecture et la relecture de ses propres travaux avec une assiduité peu commune et en prêtant spécialement attention aux mécanismes de réception de chaque publication. C'est pourquoi, en suivant Louis Marin une fois de plus, on peut distinguer trois " pluralités » qui feront de tout « recueil » une unité épistémologique (bien que toujours en dispersion latente) : ses plans de cohérence, ses réseaux de problématiques et ses directions de sens ou de recherches ${ }^{27}$. De ce point de vue, l'histoire de chaque "recueil » fabriqué par Michel de Certeau rendrait compte d'une transformation particulière à partir de laquelle on retrouvera des conceptions différentes de lecture et d'écriture et une manière de plus en plus discrète de regarder son propre passé : un monde de permissions et d'interdictions qui régira l'« ordre des livres ${ }^{28}$ et tentera d'établir un chemin possible de lecture.

\section{Pertinences et maniement des sources}

En tout cas, soit en raison de l'impossibilité naturelle de suivre les traces de tous les ouvrages lus par Michel de Certeau, soit du fait de l'énorme difficulté d'appréhender une pratique de lecture individuelle ou en communauté commandée par une suite de gestes, d'habitudes ou de choix, il est vrai que son histoire matérielle comme lecteur représente l'une des facettes peut-être les plus inexplorées des études certaliennes ${ }^{29}$. Il est indéniable que la biographie intellectuelle de François Dosse, par exemple, a bien valorisé les éléments de sa trajectoire donnant un sens particulier aux auteurs que de Certeau a lus ${ }^{30}$. Toutefois, au-delà des questions "doxiques " ${ }^{31}$, les récits biographiques sur la vie des intellectuels retiennent souvent un processus créatif en dehors des voies matérielles de la réception et de la pratique lectrice en elle-même. Ainsi, loin de contempler la matérialité des textes lus, les dimensions empiriques de la lecture ne sont souvent conçues que comme une enquête sur des idées ${ }^{32}$. Négligée en tant qu'objet autonome et considérée longtemps comme tributaire du travail scripturaire, la lecture est souvent placée à côté des enjeux de déchiffrement, comme une activité se rapportant uniquement aux tâches de compréhension du texte ou même aux propriétés particulières d'interprétation. Bien que ces conditions fassent inévitablement partie de tout processus savant de création, il est certain qu'elles correspondent à un « second moment » de la production des idées.

Il existe donc une expérience matérielle préalable et plus insaisissable liée essentiellement au contact avec l'objet imprimé dont la temporalité se veut d'abord 
double. Le premier temps, objet particulier de la sociologie, l'histoire de l'édition et la bibliographie matérielle, est lié au maniement de l'usage, aux politiques éditoriales qui, de manière souterraine, traversentla configuration esthétique d'un livre ou d'une revue, aux formes dont ce savoir a été organisé comme paratexte et aux possibilités de la circulation commerciale et d'accessibilité du public. Tel que le remarque Paul Zumthor,

la lecture n'est ni un acte séparé ni une opération abstraite [...]. À tous les horizons se dessinent les mouvements d'une désaliénation à long terme du verbe humain ; mouvements où, de crise en crise, ne cessent de se surmonter les contraires ${ }^{33}$.

C'est pourquoi un lecteur non seulement « lit » un texte, mais il entre en contact avec une reliure, un papier et une typographie particuliers sur lesquels ou à partir desquels se construit un mécanisme que l'on pourrait appeler, en termes de Gérard Genette, "palimpseste ${ }^{34}$ : c'est là le second temps. Toute lecture devient de cette façon un processus d'appropriation dans lequel on met à l'épreuve différentes formes de perception intellectuelle - et non seulement de réception - ancrant cet usage liminaire dans un premier accès manœuvrable. Ainsi, dans le contexte de notre recherche, on entendra le terme lecture comme une pratique empirique, sociale et historique fabriquée collectivement à l'intérieur de plusieurs communautés interprétatives ${ }^{35}$ souvent délimitées par leur propre représentation du monde mais dont les frontières sont toutefois poreuses.

Pour construire le parcours de Michel de Certeau en tant que lecteur nous utilisons essentiellement sa production écrite et publiée. Il faut souligner pourtant que bien que son écriture soit la principale source d'information en ce qui touche notre recherche, il est vrai qu'elle n'est pas la seule ni la plus digne de foi. Au demeurant, toute intention d'exhaustivité dans ce domaine risque de frôler l'utopie: tout d'abord, parce que la lecture en tant qu'acte, pratique ou simple réception continue d'être en soi un phénomène aussi rebelle que secret et difficile à limiter en termes d'observation empirique, et plus précisément, parce que derrière les lectures visibles de Michel de Certeau, circuleront d'autres que, pour une raison ou pour une autre, il n'a pas mentionnées : soit pourleur manque de pertinence dans un travail particulier, soit par une opération de "censure» imposée ou volontaire, soit parce que le degré d'appropriation et de sélection d'une idée est arrivé à un haut niveau d'assimilation difficile à constater, soit parce qu'il les a oubliées. Comme le souligne Pierre Bourdieu,

les déclarations concernant ce que les gens disent lire sont très peu sûres en raison de ce que j'appelle l'effet de légitimité : dès qu'on demande à quelqu'un ce qu'il lit, il entend : qu'est-ce que je lis qui mérite d'être déclaré ?36.

19 Ainsi, bien que la citation, par exemple, prétende illustrer, indiquer un renseignement complémentaire ou légitimer une idée et non pas tout ce qui est omis soit le produit d'une tactique scientifique de légitimation, il est vrai que sa pratique se construit à partir de la hiérarchisation tacite de toutes ces lectures et toutes, d'une manière ou d'une autre, se soumettent à un contrat qui déterminera saposition dans le texte. Dans ce sens, la citation, selon Antoine Compagnon,

loin d'être un détail du livre, un trait périphérique de la lecture et de l'écriture, représente un enjeu capital, un lieu stratégique et même politique dans toute pratique du langage, quand elle assure sa validité, garantit sa recevabilité ou au contraire les réfute ${ }^{37}$.

20 De même, ce jeu obéit aussià une stratégie éditoriale dont la politique impose une formalisation des lectures qui doivent être exposées ou non. De sorte que le fait d'utiliser les textes de Michel de Certeau pour évoquer ses propres pratiques de lecture exige 
d'assumer ce double aspect inévitable : d'une part, les conditions de circulation des sources explicites et, d'autre part, l'existence cachée de toutes ces lectures qu'il n'a pas confessées. En somme, malgré les efforts des différentes théories sur la construction d'un lecteur autonome, distinct de l'écrit, il est certain que le principal support de tout accès à la pratique continue d'être la graphie même ${ }^{38}$.

\section{Les résistances de l'objet}

Par ailleurs, il faut compter aussi sur une autre particularité certalienne bien attachée à cette expérience matérielle de lecture et d'écriture : la façon « dépouillée » de concevoir non seulement son profil intellectuel, mais aussi son incursion dans chacun des espaces du savoir où il a transité. Il est bien su que Michel de Certeau a souvent évité toute identification emprisonnée par les classements épistémologiques ainsi que la filiation que les institutions religieuses ou universitaires pourraient lui offrir. D'une certaine manière, il a "échappé » systématiquement à toute "identité " sociale, professionnelle ou épistémologique, autrement dit, à tout titre de permission (de toute « loi ») qui assurerait la démarche de son parcours et réglerait les enjeux de ses recherches. C'est pour cela qu'il a préféré être appelé « voyageur » ou tout au plus " historien », terme dont la mobilité lui fournissait une liberté intellectuelle dans le temps et dans l'espace délimitée uniquement par ses propres « arts de faire $"^{39}$. Ainsi donc, en construisant une représentation de luimême, de Certeau a avoué en 1970, année cruciale de son itinéraire intellectuel,

je suis seulement un voyageur. Non seulement parce que j'ai longtemps voyagé à travers la littérature mystique (et ce genre de voyage rend modeste), mais aussi parce qu'ayant fait, au titre de l'histoire ou de recherches anthropologiques, quelques pèlerinages à travers le monde, j'ai appris, au milieu de tant de voix, que je pouvais seulement être un particulier entre beaucoup d'autres, racontant quelques-uns seulement des itinéraires tracés en tant de pays divers, passés et présents, par l'expérience spirituelle ${ }^{40}$.

Encore une fois, «l'union dans la différence » devient la condition de possibilité d'un encadrement symbolique ainsi que de sa «forme ». Néanmoins, bien qu'il soit légitime de prendre cette révélation comme une représentation de soi-même, il est nécessaire, pour la penser, de s'éloigner d'elle et d'examiner historiquement ses fondements. Même si dans chaque penseur il en existe plusieurs, qui interviennent tous dans le processus créatif, il s'agit d'un aveu certainement poétique qui suggère plus qu'il n'énonce et qui est par ailleurs propre à quelques autres intellectuels inclassables du XXe siècle comme Walter Benjamin, Maurice Blanchot, Michel Foucault ou Louis Marin. À partir de là, de Certeau paraît non seulement avoir considéré comme sienne la difficulté traditionnelle des sciences sociales à délimiter leurs propres frontières épistémologiques, mais semble avoir ébauché aussi les formes de sa "mission intellectuelle » au dedans et en dehors de la Compagnie de Jésus. En effet, même si l'«identité » ignacienne ne peut, à elle seule, expliquer la pluralité d'un parcours dont l'imbrication est assez complexe, il ne faudrait pas négliger sa permanence dans le cadre d'un Ordre auquel, malgré les conjonctures historiques peu ou prou conflictuelles, il restera engagé de son vivant, soit comme écrivain dans des publications du réseau jésuite, soit comme membre actif et solidaire d'un projet spirituel à longue échéance. À cet égard, le christianisme en tant que système de croyances, de pratiques et de langages, "vécu» et «lu» à la lumière de cette spiritualité, sera l'un de ses modèles d'intelligibilité les plus perdurables ${ }^{41}$. Comme l'a exprimé Luce Giard : 
La question de Dieu, de la foi et du christianisme n'a cessé d'habiter Michel de Certeau. Elle est à la source d'une impossibilité à se satisfaire d'un seul type de savoir, d'où ce parcours méthodique de disciplines (langues anciennes avec une prédilection pour le grec, histoire, philosophie et théologie au temps des études et des diplômes ; plus tard, par manière de compléments, linguistique, psychanalyse, anthropologie et sociologie) ${ }^{42}$.

C'est cette sorte de philosophie en et $d u$ mouvement qui le mènera à construire une pensée dont la cohérence trouvera son principal lieu matériel dans une pratique furtive de la lecture et d'une écriture profondément nomade.

Quant à la visibilité de ce mouvement, il est important de ne pas confondre la «forme » avec le « contenu ». Bien que la dissémination matérielle de son œuvre soit étroitement associée aux enjeux d'une pensée " traversière » et à la quête des interstices dans et entre les disciplines, il est vrai qu'elle a conservé une organisation souterraine continue et durable. À ce sujet, au sein de ce que l'on pourrait appeler informellement un " mouvement anglophone » des études certaliennes ${ }^{43}$, à la fin des années quatre-vingtdix a surgi un débat important mais silencieux entre le théoricien anglais Jeremy Ahearne et l'australien Ian Buchanan à l'occasion de la parution de l'ouvrage du premier. Selon Ahearne, de Certeau

n'était pas intéressé à produire une construction doctrinale systématique, pas plus qu'il ne s'est lui-même érigé en tant que gardien d'une réserve érudite [...], sa stratégie intellectuelle consistait précisément à avoir le souci de discerner et de faire une éthique et un espace esthétique pour des formes particulières d'interruption. Son travail a été conçu comme une réponse continuelle à une série d'appels et de sollicitations qui lui ont été adressées directement ou indirectement par d'autres. À la lumière de cela, je ne vais pas moi-même tirer un système d'interprétation du travail de Certeau ${ }^{44}$.

Face à cela, Buchanan a répliqué vivement à travers un essai et une recension de l'ouvrage ${ }^{45}$, notamment dans le cadre de son propre travail, publié quatre ans plus tard :

Mon point de vue est que nier l'existence d'une thèse primordiale s'étendant sur la totalité d'une carrière, à la limite même d'ignorer la consistance de la méthode et la durabilité d'un substrat épistémologique, est simplement une autre façon d'éviter la question de la religiosité chez de Certeau ${ }^{46}$.

Il convient de signaler d'abord qu'à la différence de l'ouvrage d'Ahearne qui a travaillé sur le corpus certalien ad intra et dans sa langue d'origine, celui de Ian Buchanan est concentré essentiellement sur les versions anglaises et ne s'attache pas à une perspective d'ensemble. En revanche, son objectif est d'établir les liaisons théoriques ad extra que l'œuvre certalienne partage avec le post-modernisme et la pensée de Lacan, Greimas, Deleuze, Merleau-Ponty et Walter Benjamin. Tout en reconnaissant cette différence, il faut noter que, bien que Michel de Certeau n'ait pas construit du tout un «doctrinal edifice ${ }^{47}$, cela ne l'a pas empêché de bâtir un "système de pensée " fortement consistant, homogène mais « au pluriel » tout au long de son œuvre et ancré dans un " modèle interprétatif que constitue et vérifie une pratique scientifique, c'est-à-dire une organisation cohérente des procédures interprétatives ${ }^{48}$. En tout cas, Buchanan fait moins une critique de l'unité de l'œuvre certalienne (confirmée, à plusieurs reprises, par Jeremy Ahearne) qu'il n'exprime le souci pertinent d'envisager le parcours de Michel de Certeau tout entier au moment d'établir l'origine et la continuité de sa pensée, surtout par rapport à sa première époque de production intellectuelle dans la Compagnie de Jésus ou, comme il le dit lui-même un peu vaguement, à l'« issue of religiosity »49. Malgré cela et bien qu'Ahearne ne fasse qu'une légère allusion à ce passé (ainsi que Buchanan lui-même 
dans son ouvrage), son but est loin de vouloir refuser l'existence de cette première période: il s'agit d'une option, ou plutôt de la limite obligée d'une recherche qui interroge l'œuvre certalienne à partir des années soixante-dix et d'une analyse théorique essentiellement conceptuelle en dehors de toute préoccupation religieuse ou encore historiographique.

En réalité, c'est le point d'observation qui devrait être changé ou, du moins, déplacé. Tout d'abord parce qu'il ne s'agit que d'un débat développé au sein d'un bibliocentrisme qui élude la nature matérielle de la fabrication de l'œuvre certalienne, et tient compte uniquement (ou presque) de la présence de livres publiés. Ce problème est encore plus accentué chez Buchanan qui n'a consulté que les versions traduites disponibles en anglais. Certes aucun de deux théoriciens n'ignore cette question et leurs travaux représentent un effort considérable pour saisir la pensée certalienne à partir de l'« architecture conceptuelle » (et synchronique, disons-le) de ses « figures » récurrentes (chez Ahearne), ou de la mise en contexte de son œuvre dans le cadre des «cultural studies ${ }^{50}$ et du postmodernisme (chez Buchanan). Mais il est certain que les ouvrages de Michel de Certeau continuent à y apparaître comme des «blocs» livresques bien compacts dépourvus de toute matérialité plurielle à l'intérieur ${ }^{51}$. Au-delà de toute rhétorique, comme nous l'avons dit, il ne s'agit pas d'une affaire accessoire ou du côté ornemental des effets d'une recherche, mais d'une « surface » indispensable à l'heure de lire l'œuvre certalienne, et sans laquelle, à notre avis, il n'est pas possible d'expliquer, précisément, le lieu où se fondent "les formes particulières d'interruption " ou «la consistance de la méthode et la durabilité d'un substrat épistémologique ». Ce sont là deux traits qui, au fond, participent des «formes » et du «contenu » de sa pensée sans s'opposer. En bref, ce sera sur ce lieu empirique que reposera une bonne partie de l'intelligence de la pensée certalienne. Ainsi, malgré son refus de s'enfermer dans une catégorie disciplinaire, il est vrai que si de Certeau a eu un métier perdurable et au même temps pourvu d'une expérience multiple tout au long de nombreuses publications, ce métier fut celui d'écrivain de "textes brefs " ${ }^{52}$ : c'est là une économie d'écriture qui le rapproche, une fois de plus, des «arts de faire » borgésiens. Tout en reconnaissant un parcours bien visible à partir de la publication de ses ouvrages les plus diffusés, un bibliocentrisme trop fermé ne nous conduirait que vers la formulation hasardeuse d'un canon qui, en vérité, ne coïnciderait pas du tout avec le type d'œuvre que de Certeau a proposé. En somme, quoique chacun de ses ouvrages rende possible un aperçu sous forme de synthèse, ceux-ci ne suffisent pas à expliquer d'où proviennent ses idées les plus lointaines et les enjeux de chaque réemploi, de quelle manière il les a façonnés et quelles ont été les singularités de l'ancienne et de la nouvelle réception de leur espaces de publication. C'est par-là que la lecture de soi-même devient tout un outillage à dévoiler, du moins partiellement, cet enjeu.

\section{Relire le parcours certalien}

Par ailleurs, les observations de Ian Buchanan sur l'œuvre de Jeremy Ahearne comportent un deuxième point pertinent qui ne devrait pas être négligé : le besoin d'un suivi chronologique du parcours de Michel de Certeau à travers ses écrits. Non sans risque, Ahearne est en tout cas bien explicite par rapport à son choix :

J'ai fait une analyse thématique, en traitant mon corpus comme s'il s'agissait d'un ensemble synchronique tout en introduisant des nuances diachroniques. Cela 
semble le moyen le plus efficace pour introduire le lecteur dans un espace limité, à

la fois, par l'étendue et la complexité enrichissante de la pensée de Certeau ${ }^{53}$.

Même si elle n'échappe pas complètement à la logique atemporelle suivie par le "mouvement anglophone", cette détermination pourrait être plutôt mise en relation avec la position qu'occupe Interpretation and Its Other dans le cadre de l'historiographie des études certaliennes et à l'absence à ce moment-là d'une biographie de Michel de Certeau qui organisait son énorme production écrite en accord avec les changements de sa vie intellectuelle ${ }^{54}$. À cet égard, on pourrait dire que si la "Bibliographie complète » établie par Luce Giard (à côté de nouvelles éditions établies et des travaux qu'elle anima depuis 1987) marque le premier moment des études certaliennes, et si le second correspond à ce "mouvement anglophone» dont l'ouvrage de Jeremy Ahearne représente le point culminant, il est indéniable que le travail biographique publié par François Dosse en 2002 ainsi que ses recherches collectives autour de la figure de Michel de Certeau ${ }^{55}$ constituent un troisième grand moment que l'on pourrait appeler « courant français » ${ }^{56}$. À notre avis, il existe trois principales nouveautés propo-sées par la biographie de François Dosse. En premier lieu, quant aux sources utilisées, la récupération du versant épistolier de Michel de Certeau - particulièrement pour la première époque de son parcours - se veut un élément inédit dans les études de son œuvre ${ }^{57}$.En second lieu, la biographie souvent nourrit une bifurcation qui se veut essentielle à l'heure de penser la nature de sa démarche : l'énorme réseau social et intellectuel que de Certeau avait construit de son vivant et l'association par contraste qu'il entretint avec les institutions, soit ecclésiastiques, soit universitaires ${ }^{58}$. Enfin, et dans un autre ordre d'idées, il est certain que ce travail a représenté l'installation et la légitimation définitives de la figure certalienne dans une nouvelle communauté de lecteurs : le public. Écrite dans un langage accessible (mais sans tomber dans la vulgarisation extrême), publiée sans lourdes annexes bibliographiques susceptibles de faire fuir le lecteur non spécialiste, et organisée à partir d'essais " consultables » de façon individuelle et thématiquement " identifiables », la "biographie blanche ${ }^{59}$ de François Dosse non seulement fut l'objet de recension dans des revues spécialisées en France et à l'étranger, mais aussi dans les principaux journaux et magazines littéraires français. Il s'agit d'un genre de réception sans précédent dans les études certaliennes. D'ailleurs, attaché à la logique de l'histoire du temps présent, Dosse a utilisé un nombre important de témoignages oraux que lui-même a recueillis auprès d'amis et de collègues de Michel de Certeau. Ainsi, c'est la parole de l'autre qui se veut l'une des principales voies d'accès à la connaissance de sa figure. Il s'agit d'une expérience méthodologique en soi, d'une valeur testimoniale incontestable mais qui, toutefois, doit être prise avec une certaine précaution puisque François Dosse a fait de la mémoire un emploi particulier en utilisant les souvenirs des personnalités interviewées comme des sources documentaires sans fissures ${ }^{60}$. Néanmoins, son ouvrage contient, à notre avis, une autre difficulté un peu plus accusée : la scission "événementielle» que l'auteur suggère tout au début de son ouvrage avec "L'entrée en religion » et "L'entrée en modernité » à partir des évènements de 1968. Nous nous demanderons à cet égard si «la prise de parole» établit effectivement une authentique rupture dans l'itinéraire intellectuel certalien ou plutôt une continuité. Bien que Dosse ait suivi les affaires religieuses de Michel de Certeau avec un soin particulier tout au long de la biographie, il faut noter, néanmoins, que c'est l'image d'un de Certeau "classique » et à peu près "sécularisé » qui semble, encore une fois, phagocyter la spécificité de sa première époque. À cet égard, notre hypothèse suit le sens inverse : ce n'est pas le dernier de Certeau qui devrait éclairer le « premier » mais, justement, le contraire. 


\section{À la recherche du « premier » Michel de Certeau} 1943-1970 de sa production intellectuelle qui, à notre avis, est fondamentale pour comprendre non seulement les formes de sa pratique de lecture et les origines des « arts de braconner ", mais aussi pour s'enquérir de la manière dont Michel de Certeau est devenu une figure essentielle dans le paysage des sciences humaines. Étant donné que cette première période concerne une activité intellectuelle produite essentiellement dans le cadre général du monde religieux (mais dont les bords seront de plus en plus poreux), et de la Compagnie de Jésus en particulier, nous avons eu recours aux nuances méthodologiques proposées par l'historien Étienne Fouilloux dans son histoire intellectuelle et spirituelle de la pensée catholique française durant la première moitié du $\mathrm{XX}{ }^{\circ}$ siècle ${ }^{61}$. Cela signifie, en premier lieu, avoir pour but :

une histoire des idées revitalisée, qui ne se contente plus d'épingler dans son herbier une collection de systèmes desséchés, mais qui restitue leur tissu nourricier et se préoccupe de leur réception,

Et, d'autre part,

une histoire des intellectuels qui dépasse la description des parcours et des sociabilités pour s'intéresser de près aux liens qu'ils entretiennent avec les énoncés eux-mêmes ${ }^{62}$.

À partir de ce qui précède, nous tentons de faire une histoire des idées de Michel de Certeau en tant que lecteur, "façonneur » de lecteurs, et artisan d'une «théorie » de la lecture, et également en tant qu'intellectuel tout court. À ce sujet, même si le contexte religieux dans lequel il développa sa première production écrite a sa propre spécificité, et que sa reconstruction s'impose comme une tâche nécessaire, cela n'impliquera pas que la production de cette époque soit devenue une affaire exclusivement religieuse. Qu'il s'agisse de la conception que la Compagnie avait de l'activité intellectuelle au dedans et en dehors de l'Ordre, ou de la manière ad extra de diffuser la pratique et la "théorie " d'une spiritualité singulière, l'histoire intellectuelle des jésuites français du xxe siècle (y compris celle de Michel de Certeau) ne peut pas être entendue à la manière d'une «île " ou enfermée dans une historiographie purement religieuse. Le fait de conserver des contours bien définis n'empêche pas qu'elle fasse partie d'une histoire culturelle plus vaste, d'autant plus que les frontières (symboliques et empiriques) de la spiritualité ignacienne ne reposent pas sur la base d'une observance dogmatique et que c'est l'intégration au monde qui détermine en fin de compte la dialectique de ses mouvements. Il y a là, précisément, l'une des clés pour comprendre l'entrée de Michel de Certeau dans la Compagnie, ainsi que la nature du «réemploi» de textes et de concepts, et la façon dont il rassemblera son propre passé scripturaire.

D’ailleurs, si Ian Buchanan a souligné le besoin de récupérer la totalité du parcours certalien, c'est l'historien anglais Peter Burke qui a remarqué l'importance de sa première époque en mettant l'accent sur un type de réemploi lié à un " art of re-interpretation » :

L'analyste du processus du réemploi était lui-même un grand maître de l'art. Certeau avait un don remarquable pour la pensée analogique. Comme il devient de plus en plus intéressé par les problèmes séculaires, il les a approchés en faisant usage de notions qui ont déjà été utilisées à la fois par lui-même et par d'autres dans le domaine religieux en les adaptant à de nouveaux buts. Son appareil

Les Cahiers du Centre de Recherches Historiques, 44 | 2009 
conceptuel, ou selon la célèbre phrase de l'historien Lucien Febvre, son outillage

mental, a été récupéré de la religion et traduit en termes profanes ${ }^{63}$.

traduction de concepts du « religious domain » aux espaces du savoir « séculier », il est certain qu'une bonne partie de ce que nous appellerons la grammaire jésuite (souvent ouverte aux successifs réemplois à partir de sa propre tradition littéraire, mais aussi du monde des idées extra-religieuses) est à l'origine de plusieurs chemins sémantiques suivis par l'univers conceptuel de Michel de Certeau. Tel sera donc le cas du «braconnage». Tout bien considéré, il faut remarquer que ces lignes de continuité ne comporteront pas de traces directes, mais elles auront de multiples arrêts, des déviations, et des reconstructions qui feront des anciens termes un passé lointain, voire inattendu pour de Certeau lui-même. En tout cas, ce sont-là les enjeux d'un lecteur en " palimpseste » dont la marche fera de lui autant un pèlerin qu'un nomade. C'est ainsi qu'entre 1950 et 1970, Michel de Certeau eut l'habitude d'accéder à une sorte de lecture en communauté qui établissait différents degrés de "discernement ", une pratique construite non seulement à partir d'une tradition propre à la Compagnie de Jésus, mais aussi au sein des milieux académiques auxquels il participait. Sa mise au point, en somme, renvoie à un autre de Certeau qui devra lire en solitaire et ériger la direction de sa propre herméneutique à travers ses mouvements comme lecteur.

\section{Débroussailler les imprimés}

Ainsi, cette pratique comporte, d'une part, une itinérance de pèlerinage, un type de mouvement qui fait essentiellement partie de la cosmovision jésuite ${ }^{64}$. Véritable exercice d'ascèse transformante, le cheminement du pèlerin est, avant toute chose, une épreuve où la prière et la méditation deviennent non seulement une étape fondamentale au cours d'un long voyage, mais aussi un renoncement corporel, émotionnel et spirituel ${ }^{65}$. Dans ce contexte, étant donné que le pèlerinage se veut l'acte de dépasser les frontières et d'élargir l'horizon jusqu'à en transformer la vie spirituelle selon la volonté divine ${ }^{66}$, on peut dire que la lecture deviendra aussi une recherche de l'absolu. En tout cas, dans les termes d'Alphonse Dupront,

ce qui marque le fait pèlerin, ainsi manifesté dans le double quantitatif du temps et de l'espace, d'une ambivalence plus harmonieusement intense que d'autres comportements collectifs : celle d'une part d'un acte personnel engagé, voire responsable, et d'autre part d'un assouvissement, ce vivant, de besoins inhérents à la condition humaine. Autrement dit, en lui et de façon éminemment expressive, vécu historique et ordre anthropologique intimement liés ${ }^{67}$.

Précisément, dans les chantiers multiples de la seconde après-guerre, Michel de Certeau sera un jésuite à la recherche d'un espace double aussi sûr que symbolique : l'édification et diffusion d'une nouvelle spiritualité "vécue", et les origines historiques de la « première » Compagnie. C'est le lecteur pèlerin qui marche vers cet « ailleurs » face auquel il exprimera le besoin de récupérer un espace "autre ", et pour cela, cette "fixation spatiale" sera sa principale empreinte puisqu'il n'y aura pas d'errance dans ce pèlerinage, mais un « lieu » dont le terme sera assuré et connu au préalable ${ }^{68}$. Ainsi, lire en pèlerin sera concevoir la pratique à la façon d'une traversée de l'objet imprimé où le lecteur sera toujours, dans ce sens, un «étranger» qui part au loin à travers une prospection incessante qui l'emmène en exil. C'est pour cela qu'à l'instar de sa nature fuyante, toute lecture est déjà une pratique de l'altérité en elle-même. Ce processus 
s'organisera à partir d'une dimension définie de lectures plutôt hiérarchisées qui comprendra, pour tacite qu'il soit, un système de libertés et d'interdictions relatives qui met en ordre tout ce qui ne peut pas ou qui ne doit pas être lu. Néanmoins, bien qu'il soit vrai qu'en tant que lecteur son inscription sociale imposait à ses manières de lire un premier ordre de sens construit collectivement par la Compagnie, celles-ci ne se trouvent pas pour autant habitées uniquement par un intérêt théologique ou spirituel. À ce sujet, on peut distinguer un trait caractéristique de cette première époque de pratique pèlerine: il s'agit d'un rapport anthropologique et historique à une spiritualité qui, d'abord, sera essentiellement ignacienne. Ce sont là deux horizons gnoséologiques qui faisaient partie de son premier univers conceptuel longtemps avant l'avènement du de Certeau « classique ».

Ce parcours initial de lecture conserve ainsi une trace religieuse claire et une préoccupation marquée par les enjeux du christianisme. C'est à partir de celles-ci que de Certeau s'empressera de mettre l'accent sur une somme de fissures que, plus tard, il étendra au champ des sciences humaines. C'est là que le lecteur nomade fera ses premières apparitions intermittentes moyennant un jeu où l'exposition et l'effacement d'un savoir "étranger » au discours religieux dénoteront la marque d'une autre présence. Si c'est la quête de nourriture qui anime le déplacement du nomade, il faut dire que l'expérience de la «chasse» sera à l'origine de ses mouvements de lecteur à travers des infiltrations lentes ou bien des invasions abruptes. En tout cas, bien que le pèlerinage et le nomadisme comprennent deux temporalités différentes de lecture, ils seront impliqués dans un même mouvement diachronique qui se déroulera simultanément. Orienté par différentes traces de l'écrit, de Certeau s'appropriera du dedans plusieurs espaces du savoir, selon de multiples manières, mais sans prétendre s'installer dans l'un d'entre eux. Il faudra insister sur la notion d' "espace de savoir » parce qu'il n'abordera pas, pendant cette époque du moins, des "disciplines", mais leurs espaces internes en quête de figures ou d'outils capables de rendre intelligible ou d'éclairer une problématique spécifique, et cela à partir d'un choix de ressources mobiles qui opèreront souvent de façon réversible ${ }^{69}$. C'est pour cela que d'un imprimé à un autre, son circuit nomade sera subordonné à une cartographie de plus en plus particulière d'où proviendra, justement, l'autonomie que chacun de ces espaces aura dans le futur ${ }^{70}$.

En tout cas, il faut rappeler que ni le pèlerinage, ni le nomadisme ne seront des déplacements "purs » dans les territoires des imprimés. Ils partagent un chemin commun en ce qu'ils interagissent l'un avec l'autre en donnant lieu à une pratique plus complexe : les arts de braconner. Tout au début de son parcours intellectuel, et à la façon d'une poétique, de Certeau fabriquera un "art de lire» bien personnel situé à la confluence de la marche du pèlerin et de l'incursion du nomade. Tout texte deviendra ainsi un espace de tension sur lequel se déploieront deux façons de lire étroitement imbriquées : une "fixation spatiale" de l'écrit attachée à la certitude d'avoir un emplacement assuré au terme de la lecture, et, en même temps, le besoin d' "échapper " à celui-ci à travers le désir de continuer d'avancer pour saisir un objet le plus souvent «absent ». À ce sujet, et en tant que membre d'une nouvelle génération intellectuelle, Michel de Certeau accueillera dans sa propre pratique la transition de deux représentations différentes de la connaissance: si, d'une part, il gardera une tradition d'appropriation propre à ses maîtres par le biais de l'accumulation érudite, d'autre part, il fera de toute saturation savante un usage entièrement disséminé en la soumettant à des conditions changeantes de mobilité périodique. Les premiers pas de son braconnage 
porteront ainsi la marque d'une démarche circonscrite historiquement dans le cadre d'une volonté ininterrompue de "progrès spirituel » afin de rendre lisible une nouvelle pratique de la spiritualité.

D'ailleurs, face à notre décision de nommer "arts de braconner" les pratiques certaliennes de la lecture, on pourrait assez justement nous objecter qu'il ne s'agit que d'une autre façon de placer au premier plan, encore une fois, le de Certeau «classique ». Certes, mais en partie seulement. Il est vrai que nous employons un terme assez tardif chez lui et, sans aucun doute, associé au de Certeau le plus diffusé. Cependant, il est certain aussi que l'on peut repérer différentes traces de sa pratique du braconnage dès ses premiers textes publiés. À cet égard, la pratique du braconnage ne sera qu'une autre manière de « débroussailler » les imprimés : de Certeau non seulement tracera différents chemins en écartant les obstacles afin de capturer la matérialité des idées, mais encore il fragmentera les usages de cette pratique. Ses manières de s'adresser aux lecteurs ou même de produire et de "façonner » ces derniers, l'attention portée aux modes de lire de ses « créatures textuelles ", la pratique de la " lecture de soi-même » (lorsqu'il se dispose à lire ses propres écrits pour mener à bien un travail de "réemploi » et construire un ouvrage), ou encore son usage de la citation qui révèle la zone évasive d'un «lecteur universel ", montrent, sans aucun doute, que de Certeau a réfléchi sur la nature de la lecture et sur le rôle du lecteur longtemps avant la parution de son article dans la revue Projet en 1978. Comme l'a observé Daniel Frey au sujet de la naissance de l'histoire de la lecture comme objet d'étude,

il est historiquement avéré que la lecture, en tant qu'activité du sujet lecteur, est le fruit d'un affranchissement progressif de l'opération de déchiffrement. Elle ne s'est imposée tardivement comme objet de recherche que parce qu'elle-même s'est constituée graduellement comme une activité à part entière ${ }^{71}$.

Ainsi, il faut remarquer que, dans le cadre de son œuvre, le terme "braconnage » se voudra plutôt une mise au point qui synthétise un long parcours intellectuel où la pratique de la lecture, bien entendu, a occupé un espace central. Une sorte de "somme » qui pourrait s'appliquer à la production de ses deux derniers grands ouvrages, L'invention du quotidien (1980) et La Fable mystique (1982) où, d'une manière ou d'une autre, le de Certeau lecteur objective et réemploie un ancien espace de recherche à travers un exercice poétique qui se rend à l'évidence : c'est lui-même qui est devenu un « autre » et, après tout, son propre braconnier ${ }^{72}$.

Précisément, le dernier essai qu'il a écrit pourrait se concevoir comme une fable théorique qui fonde cette évidence symbolique. Il s'agit de «L'expérimentation d'une méthode : les Mazarinades de Christian Jouhaud », un texte écrit au début de janvier 1986 - peu de jours avant sa mort - et publié quelques mois plus tard dans la revue Annales ESC ${ }^{73}$. Comme son titre l'indique, il s'agit de la recension de Mazarinades: la Fronde des mots ${ }^{74}$, un ouvrage devenu aujourd'hui un classique que le jeune Jouhaud avait publié en février 1985 et où il construisait une nouvelle méthode de lecture pour s'enquérir de la structure spécifique des mazarinades, c'est-à-dire, l'ensemble de libelles, de pamphlets et de chansons qui circulaient à Paris vers le milieu du XVII siècle pendant la Fronde contre le Cardinal Mazarin. À cet égard, cet article de Michel de Certeau renvoie à l'une de ses pratiques de lecture les plus explicites, autrement dit, à l'écriture de recensions bibliographiques, laquelle conserve un mérite allégorique et circulaire : un de Certeau lecteur "visible" commente une méthode de lecture historique que lui-même a contribué à édifier. Vingt ans après, à l'occasion de la réédition de l'ouvrage, Christian Jouhaud écrit une nouvelle 
préface où il dialogue avec, mais aussi répond au de Certeau de la recension qui se voulait "précis, chaleureux, mais sans complaisance»: la marque d'une généalogie resurgit encore une fois ${ }^{75}$.

\section{NOTES}

1. Cet article indique les orientations fondamentales de ma thèse de doctorat de l'EHESS consacrée à reconstruire les pratiques de lecture de Michel de Certeau, en particulier dans la première époque de son parcours intellectuel (1943-1970). À partir des marginalia laissées par Michel de Certeau dans les livres de sa bibliothèque personnelle, de son usage de la citation et de la note ainsi que de la manière dont lui-même a lu ses propres écrits pour les reprendre dans des livres qui sont autant de "recueils", j'essaie de bâtir une généalogie de la lecture comme «braconnage » telle qu'on la rencontre dès le «premier» Michel de Certeau. Je remercie mon directeur d'études, Roger Chartier, de m'avoir autorisé à publier dans les Cahiers $d u$ CRH ce premier état de mon travail, ainsi que $\mathrm{M}^{\mathrm{me}}$ Luce Giard dont les indications m'ont été essentielles.

2. Selon Louis Marin, « Traverser les signes, ce fut la préoccupation permanente et le permanent souci de ses voyages intellectuels et de ses entreprises savantes", Louis Marin, "L'aventure sémiotique, le tombeau mystique », in Michel de Certeau, Luce Giard (dir.), Paris, Centre Georges Pompidou, coll. « Cahiers pour un temps », 1987, p. 208.

3. MichelLafon, Borges ou la réécriture, Paris, Seuil, coll. "Poétique», 1990. Nous pourrions reprendre la question que Lafon se pose au sujet de la temporalité de la réécriture : «Le danger est double, de la voir partout et de ne la voir presque nulle part. Presque nulle part, c'est à confondre réécriture et citation; partout, c'est à substituer incitation à réécriture » (cf. ch. VIII «Réécriture de l'universel intertexte », p. 110). Dans le cas de Michel de Certeau, il faudra prêter attention aux textes qui furent l'objet de « réemploi », mais aussi à ceux qui restèrent dans sa publication d'origine et ne furent jamais réemployés. À cet égard, le « recueil » en tant qu'outil pour examiner une sélectivité et une "politique de la visibilité » textuelle pourrait fonctionner aussi comme un « lieu de mémoire " à l'occasion d'un changement historique de paradigme où la «mémoire» procède par «éclairage ponctuel, multiplication de prélèvements sélectifs, échantillons significatifs». Pour reprendre la belle phrase de Pierre Nora, «Le devoir de mémoire fait de chacun l'historien de soi " cf. Pierre Nora, «Entre mémoire et histoire. Le problème des lieux ", in Les lieux de mémoire I. Pierre Nora (dir.), (1984). Paris, Gallimard, coll. « Quarto », 2008, p. 23-43.

4. Irène Langlet, "Parcours du recueil ", in Le recueil littéraire. Pratiques et théorie d'une forme, Irène Langlet (dir.), 2003, Rennes, Presses Universitaires Rennes, coll. «Interférences », 2003, p. 11-18.

5. Nous prenons le terme d'après l'ouvrage d'Emmanuel Fraisse, Les anthologies en France, Paris, PUF, coll. «Écriture », 1997, p. 9.

6. Voir à cet égard les deux travaux de Jacques Le Brun, à présent capitaux pour comprendre les origines intellectuelles du travail sur les textes dans la pensée de Michel de Certeau : en premier lieu, «Le secret d'un travail » in Le voyage mystique. Michel de Certeau, Luce Giard (dir.), Paris, Recherches de Science Religieuse / Les Éditions du Cerf, 1988, p. 77-91, et particulièrement, « De la critique textuelle à la lecture du texte", Le Débat, $n^{\circ} 49$, dossier "Michel de Certeau, historien ", p. 109-116. 
7. Michel de Certeau, «Lire : braconnage et poétique des consommateurs », Projet $\mathrm{n}^{\circ}$ 124, avril 1978 (Numéro spécial intitulé «Consommateurs d'informations »), p. 447-457. Le texte fut repris avec des modifications à deux occasions de plus, in Michel de Certeau, École et cultures : déplacer les questions, Écho des conférences et séminaires d'anthropologie, semestre d'été 1978, Cahiers de la Section des Sciences de l'Éducation, "Pratiques et Théorie ", nº 13, Université de Genève, Faculté de Psychologie et des Sciences de l'Éducation, mai 1979, p. 29-44, et in Michel de Certeau, L'invention du quotidien I. Arts de faire, Paris, Union Générale d'Éditions, coll. «10-18 », 1980, chapitre XII, p. 279-296.

8. Michel de Certeau (1979), «La lecture absolue (Théorie et pratique des mystiques chrétiens : XVI ${ }^{e}-X V I I{ }^{e}$ siècles), in Lucien Dällenbach, et Jean Ricardou (Centre Culturel International de Cerisyla-Salle), Problèmes actuels de la lecture, Paris, Clancier-Guénaud, coll. "Bibliothèque de Signes ", 1982, p. 65-80.

9. Michel Foucault (1982), L'herméneutique du sujet. Cours au Collège de France (1981-1982), Édition établie sous la direction de François Ewald et Alessandro Fontana, par Frédéric Gros, Paris , Gallimard / Le Seuil, coll. « Hautes Études ", 2001, Cours du 3 mars 1982, Deuxième heure, p. 341. 10. Cf. Donald Francis McKenzie (1986), «Le livre comme forme expressive », in La bibliographie et la sociologie des textes. Traduit de l'anglais par Marc Amfreville. Préface de Roger Chartier, Paris, Éditions du Cercle de la Librairie, [hors collection], 1991, p. 25-54.

11. Cf. Luce Giard (dir.), "Le voyage mystique. Michel de Certeau», Recherches de Science Religieuse, 1988, tome LXXVI, oㅡㄹ, p. 161-262 et nº 3, p. 321-457.

12. Cf. Luce Giard (dir.), Le voyage mystique. Michel de Certeau, Paris, Recherches de Science Religieuse/Les Éditions du Cerf, 1988.

13. "Établir une bibliographie complète est une entreprise malaisée, presque autant que de dresser l'inventaire exhaustif d'une langue vivante. Cette bibliographie se contentera, plus modestement, d'être d'intention complète, en sachant l'impossibilité de l'être vraiment ", in Luce Giard, «Bibliographie complète de Michel de Certeau » (Notice liminaire), in Luce Giard, (maître d'œuvre), op. cit., p. 191.

14. Nous pourrions parler d'une "école française ", en quelque sorte fondatrice des études certaliennes et animée essentiellement par Luce Giard qui concerne une mise en portée de la figure intellectuelle et religieuse de Michel de Certeau et notamment de la construction d'un projet de récupération textuelle à deux fronts. Au premier but, répond l'organisation des colloques et des ouvrages collectifs, c'est-à-dire, celui publié par la collection « Cahiers pour un temps » du Centre Georges Pompidou (Paris, 1987), le dossier paru dans Recherches de Science Religieuse ainsi que son tiré à part, déjà cités (Paris, 1988), et les textes du colloque célébré au Centre Sèvres en 1988 et publié en collaboration avec Hervé Martin et Jacques Revel sous le titre Histoire, mystique et politique. Michel de Certeau (Paris, 1991). Quant au projet de récupération textuelle, il pourrait se subdiviser en deux parties. D'un côté, il comporte la réédition et l'éventuel établissement des grands classiques de Michel de Certeau en format de poche à l'exception de L'absent de l'histoire, déjà paru dans ce format en 1973 chez Mame dans la collection «Repères ", aujourd'hui disparue. D'un autre côté, la construction de « recueils » qui récupèrent une bonne partie des textes dispersés : si l'on excepte La prise de parole et autres écrits politiques (où sont recueillis deux livres presque entiers, La prise de parole. Pour une nouvelle culture (1968) et L'ordinaire de la communication (1984) à côté de trois textes de plus) paru au Seuil en format de poche en 1994, ce sont trois des «recueils» construits au complet par Luce Giard: les deux éditions de La faiblesse de croire, Paris, Seuil, coll. "Esprit», 1987, et « Points Essais », 2003, les deux éditions d'Histoire et psychanalyse entre science et fiction, Paris, Gallimard, coll. "Folio Histoire ", 1987 et 2002, et Le lieu de l'autre. Histoire religieuse et mystique, Paris, Gallimard/Seuil, coll. " Hautes Études ", 2005. Souvent dangereux à cause de son déterminisme latent, le terme « école » semble pourtant pertinent dans ce cas. Bien que Luce Giard ne soit la porteuse d'aucun manifeste et que l'apport «au pluriel » de chaque spécialiste (procédant le plus souvent des 
milieux historiens) à ses ouvrages collectifs ne soit qu'à titre personnel, il est vrai que son dessein comporte une morphologie esthétique particulière et bien définie ainsi qu'un travail homogène d'établissement textuel et un processus de «singularisation » de la pensée certalienne dans le cadre d'une logique historique de son parcours. À cet égard, et sans l'intention de "faire école ", il faut rappeler qu'une bonne partie des travaux sur l'œuvre de Michel de Certeau continue à être, d'une manière ou d'une autre, débitrice de son projet.

15. C'est le terme récupéré par Luce Giard dans son étude liminaire à partir de l'usage certalien : «On sait que Certeau travaillait et retravaillait la trame de ses écrits, il corrigeait et recorrigeait jusqu'au bon à tirer, il remaniait sans cesse et demeurait toujours insatisfait du résultat final. De ce procès de travail dépend son habitude constante du réemploi. Il procédait comme un peintre, par états successifs, à partir d'une première esquisse d'ensemble ou d'une série de croquis de détail », Luce Giard, (Notice liminaire), in «Bibliographie complète de Michel de Certeau » Luce Giard (dir.), op. cit., p. 193 (C'est Luce Giard qui utilise les italiques).

16. Michel de Certeau, L'invention du quotidien I. Arts de faire, ch. X «L'économie scripturaire», $\S$ « Écrire : une pratique mythique moderne », p. 235.

17. Ibid., p. 236-237.

18. Nous reprenons le terme de Jean-Pol Madou au sujet de la poétique de l'écrivain martiniquais Édouard Glissant, Jean-Pol Madou, «Edouard Glissant: Tout-monde, une poétique de l'archipel (par-delà Faulkner et Saint-John Perse) », in French Prose in 2000, Michael Bishop, \& Christopher Elson (edit.), Amsterdam, Rodopi, coll. « Faux Titre », 2002, p. 1-14.

19. Selon Gérard Genette, «Le paratexte est donc pour nous ce par quoi un texte se fait livre et se propose comme tel à ses lecteurs, et plus généralement au public. Plus que d'une limite ou d'une frontière étanche, il s'agit ici d'un seuil, ou - mot de Borges à propos d'une préface - d'un "vestibule" qui offre à tout un chacun la possibilité d'entrer, ou de rebrousser chemin ", Gérard Genette, [1987], Seuils, Paris, Seuil, coll. « Poétique », 1999, « Introduction », p. 7-8.

20. Voir à cet égard, José Emilio Burucúa, «La noción de alteridad y el caso de la historia de Ulises en el Renacimiento », Eadem utraque Europa, Buenos Aires, oํ 4/5, juin-décembre 2007, p. 191-228.

21. Michel de Certeau, L'étranger ou l'union dans la différence, Paris, Desclée de Brouwer, coll. « Foi Vivante », 1969, « Avant-propos », p. 6.

22. Michel de Certeau, L'absent de l'histoire. s. 1., Mame, coll. « Repères », 1973, « Avant-propos », p. 8.

23. Michel de Certeau, «Les révolutions du « croyable » », Esprit, $n^{\circ} 378$, février 1969, p. 190, n. 1. 24. Je remercie M. José Emilio Burucúa pour tous les renseignements liés à la figure d'Emmanuele Tesauro.

25. C'est Louis Marin qui a tracé les limites de cette notion : «Pièges de discours, soit : procédés rhétoriques, opérations argumentatives, tactiques dialogiques visant à persuader l'autre, à manipuler l'auditeur, à le réduire au silence ou à le faire croire [...]. Qui est le piégeur ? Le narrateur dissimulé dont le récit dénie la présence. Et le piégé ? Le lecteur qui croit entendre le récit des événements eux-mêmes à la faveur de cette absence et qui écoute de cette voix inaudible la sentence de la vérité même dans le fait sur la page transcrit : histoire ", Louis Marin (1978), Le récit est un piège, Paris, Minuit, coll. « Critique », 1990, p. 8. C'est l'auteur qui souligne.

26. Louis Marin, Études sémiologiques. Écritures, peintures, Paris, Klincksieck, coll. "Esthétique », 1971, « Avant-propos », p. 14.

27. Louis Marin, op ; cit., p. 14.

28. Nous pensons le terme « ordre des livres » à partir de Roger Chartier, selon lequel ce sont les trois façons de comprendre la notion: «Son premier sens désigne les opérations multiples qui rendent possible la mise en ordre du monde de l'écrit [...]. Un second sens vise l'ordre que le texte entend imposer au lecteur, que cet ordre soit celui de la lecture, impliqué par la forme du livre, celui de la compréhension, ou bien encore celui voulu par l'autorité qui a commandé, autorisé ou 
diffusé l'ouvrage [...]». Quant au troisième sens, il correspond aux livres comme réalités physiques "dans leur matérialité même, commandent la possible appropriation des discours ", Roger Chartier, Culture écrite et société. L'ordre des livres (XIVe-XVIII siècle), Paris, Albin Michel, coll. «Histoire ", 1996, «Introduction», p. 14-15. Néanmoins, dans le cas de Michel de Certeau, à l'« ordre des livres » il faudra ajouter un ordre particulier « des revues » sans lequel, il serait bien difficile de mesurer l'impact de la matérialité qui portent ses « recueils ».

29. Néanmoins, ce sont nombreuses les études qui utilisent l'idée d'un de Certeau « lecteur de » ou qui envisagent la possibilité de « lire » son œuvre autrement. À ce dernier égard, ce sont deux des revues qui lui ont consacré un numéro spécial sous le titre "Lire Michel de Certeau », la Revue de Théologie et de Philosophie (Genève), vol. CXXXVI, no 4, 2004, et la revue Zeitsprünge. Forschungen zur Frühen Neuzeit (Frankfurt), vol. XII, $n^{\text {os }} 1 / 2$, 2008. Comme exemples du premier cas, il faut mentionner l'article d'Anne-Marie Chartier et Jean Hébrard, «L'invention du quotidien. Une lecture, des usages ", Le Débat, nº 49, 1988, p. 97-108; celui de Claude Rabant, "Michel de Certeau, lecteur de Freud et de Lacan ", EspaceTemps, $n^{\text {os }} 80 / 81,2002$, p. 22-26 ; le texte de Frank Lestringant, "Lectures croisées de Jean de Léry: à propos du "Bréviaire de l'ethnologue" " in Christian Delacroix, et al., Michel de Certeau. Les chemins d'histoire, p. 55-75; et le chapitre II « À l'épreuve de l'expérience : Michel de Certeau, lecteur de Machiavel », in Serge Mboukou, Michel de Certeau. L'intelligence de la sensibilité. Anthropologie, expérience et énonciation, Strasbourg, Le Portique, coll. «Cahiers du Portique », 2008, p. 45-69.

30. François Dosse, Michel de Certeau. Le marcheur blessé, Paris, La Découverte, [hors collection], 2002.

31. Pierre Bourdieu, "L'illusion biographique », Actes de la Recherche en Sciences Sociales, vol. LXII, no 62-63, 1986, p. 69-72, et Giovanni Levi, « Les usages de la biographie », Annales, vol. XLIV, nº 6, 1989, p. 1325-1326. Voir aussi François Dosse, Le pari biographique. Écrire une vie, Paris, La Découverte, [hors collection], 2005, p. 399-437.

32. À cet égard, en pensant aux pratiques culturelles de la lecture en termes d'appropriation, Roger Chartier a souligné que «la première précaution est de ne point confondre l'étude des textes avec celle des gestes ou des pensées qu'ils sont censés produire. Constat d'évidence, mais constat souvent oublié puisque l'historien, le plus souvent, n'a pris que sur des discours où s'énoncent des conduites à imiter ou des intrigues à déchiffrer ", Roger Chartier, Lectures et lecteurs dans la France d'Ancien Régime, Paris, Seuil, coll. «L'Univers Historique », 1987, « Avantpropos ", p. 13.

33. Paul Zumthor, Performance, réception, lecture, Québec, Le Préambule, coll. «L'Univers des discours ", 1990, ch. III « Performance et lecture ", p. 68.

34. Gérard Genette (1982), Palimpsestes. La littérature au second degré, Paris, Seuil, coll. "Poétique ", 1987.

35. Stanley Fish, (1979), "Is There a Text in This Class?", in Is There a Text in This Class? The Authority of Interpretative Communities. Cambridge / Massachusetts, Harvard University Press, [hors collection], 1980, p. 303-321.

36. Pierre Bourdieu, «La lecture : un pratique culturelle. Débat entre Pierre Bourdieu et Roger Chartier ", in Roger Chartier (sous la direction de, et à l'initiative de Alain Paire) (1985), Pratiques de la lecture, Paris, Payot \& Rivages, coll. « Petite Bibliothèque Payot », 2003, p. 284.

37. Antoine Compagnon (1979), La seconde main ou le travail de la citation, Paris, Seuil, [hors collection], 2002, p. 12.

38. Nina Catach, «La graphie en tant qu'indice de bibliographie matérielle ", in La bibliographie matérielle, Table ronde présentée par Roger Laufer et organisée pour le CNRS, par Jacques Petit, Paris, Éditions du Centre National de la Recherche Scientifique, 1983, p. 115-123.

39. Cf. Roger Chartier (1986), «L'histoire ou le savoir de l'autre », in Michel de Certeau, Luce Giard (dir.), Paris, Centre Georges Pompidou, coll. « Cahiers pour un temps », 1987, p. 155-167, et repris in Roger Chartier, Au bord de la falaise. L'histoire entre certitudes et inquiétude, Paris, Albin Michel, 
coll. « Histoire ", 1998, sous le titre "Stratégies et tactiques. De Certeau et les "arts de faire" ", p. $161-172$.

40. Michel de Certeau, "L'expérience spirituelle", Christus, $\mathrm{n}^{\circ}$ 68, 1970, p. 488. Voir aussi la nouvelle édition introduite et établie par Luce Giard de l'ouvrage de Michel de Certeau (1969), L'Étranger ou l'union dans la différence, Paris, Seuil, coll. « Points Essais », 2005, 2005, p. 1-2.

41. À cet égard, le principal travail en langue française qui étudie les traces du rapport entre Michel de Certeau et le christianisme, ainsi que son éventuel rôle de "théologien ", est celui dirigé par Claude Geffré et intitulé Michel de Certeau ou la différence chrétienne, Paris, Les Éditions du Cerf, coll. «Cogitatio fidei » (1991), lequel rassemble les travaux présentés à l'occasion du colloque "Michel de Certeau et le christianisme » qui s'est tenu au Centre Thomas-More en septembre 1989.

42. Luce Giard, "Cherchant Dieu », in Michel de Certeau, La faiblesse de croire. Texte établi et présenté par Luce Giard, Paris, Seuil, coll. «Esprit », 1987, p. I.

43. En ce qui concerne la production anglophone sur l'œuvre de Michel de Certeau, nous avons préféré prendre l'idée de "mouvement » en raison de la manière hétérogène d'en aborder les topiques certaliens et de se les approprier. À la différence de l'«école française » dont la communauté inter-prétative se trouve bien attachée au cadre historique de son parcours, le «mouvement anglophone» se caractérise par la construction d'un de Certeau souvent " atemporel » lié, soit aux «cultural studies ", soit à la « critical theory » surtout à partir de la version anglaise de Arts de faire (The Practice of Everyday Life. Traduit par Steven Rendall. Berkeley, University of California Press, hors collection, 1984), mais aussi d'un recueil sans équivalent français, Heterologies. Discourse on the Other, Minneapolis, University of Minnesota Press, coll. "Theory and History of Literature », vol. XVII, 1986, organisé au complet par Michel de Certeau de son vivant et publié après sa mort (cf. Jeremy Ahearne, «Feux persistants. Entretien sur Michel de Certeau ", «D’Europe en Amérique : la circulation de l'œuvre », Esprit, nº 219, mars 1996, p. 135-136). D’ailleurs, le grand mérite de ce vrai mouvement intellectuel est l'ample diffusion de la pensée certalienne. Il démontre de même une mise à l'épreuve des processus d'appropriation lectrice telle que lui-même décrit dans son essai sur la lecture en 1978. Néanmoins, le prix de cette diffusion implique un certain abandon du «premier » de Certeau et une omission frappante sur la nature matérielle de son œuvre, une omission qui certainement et à l'exception de la recherche de Luce Giard, pourrait s'étendre à une bonne partie des études certaliennes quelle que soit son origine. Cette opération de "traduction », en somme, à laquelle de Certeau lui-même a participé, aura de fortes conséquences sur la façon de comprendre sa figure et, naturellement, de lire les enjeux de son démarrage (cf. le texte de Steven Ungar, "Réceptions et actualités américaines de Michel de Certeau », in Michel de Certeau. Les chemins d'histoire, Christian Delacroix, et al. (dir.), Paris, Complexe, coll. « Histoire du temps présent ", 2002, p. 87-98 ; François Dosse, Michel de Certeau. Le marcheur blessé, op. cit., « La réception anglosaxonne ", p. 426-440; et les différents «cercles de réception» de son œuvre dans la première partie de l'article d'Isabelle Ullern-Weite, « En braconnant philosophiquement chez Certeau. Des usages de "l'historicité contemporaine" à la réinvention ordinaire de la civilité ", Revue de Théologie et de Philosophie (Genève), vol. CXXXVI, nº 4, 2004, p. 347-357.

44. «[Michel de Certeau] was not interested in producing a systematic doctrinal edifice, nor did he set himself up as the guardian of an erudite preserve [...] his intellectual strategy consisted precisely in an endeavour to discern and to make ethical and aesthetic space for particular forms of interruption. His work was conceived as an ongoing response to a series of appeals and solicitations addressed to him directly or indirectly by others. In the light of this, I shall not myself extract an interpretative system from Certeau's work ", in Jeremy Ahearne, Michel de Certeau. Interpretation and Its Other, Stanford, Stanford University Press, coll. « Key Contemporary Thinkers », 1995, « Introduction », p. 3. 
45. Cf. Ian Buchanan, «From Work to Textbook », Social Semiotics, vol. VI, no 1, 1996, p. 147-155, et « Heterophenomenology, or de Certeau's Theory of Space », ibid., p. 111-132.

46. «My point is that denying the existence of an overarching thesis spanning the entirety of a career, to the extreme even of ignoring a consistency of method and a durability of epistemological substrate, is really just another way of avoiding the issue of de Certeau's religiosity ", in Ian Buchanan, Michel de Certeau. Cultural Theorist, London, Sage Publications, coll. «Theory, Culture et Society », 2000, ch. I « The Plane of Immanence », p. 12.

47. Sur les possibilités de penser une « doctrine » chez Michel de Certeau par rapport à son cher concept «formalité des pratiques», voir l'article de Philippe Büttgen, «Le contraire des pratiques. Commentaires sur la doctrine de Michel de Certeau», Zeitsprünge. Forschungen zur Frühen Neuzeit (Frankfurt), vol. XII, $\mathrm{n}^{\text {os }} 1 / 2$ consacrés à « Lire Michel de Certeau. La formalité des pratiques », Philippe Büttgen et Christian Jouhaud (édit.), 2008, p. 69-97.

48. C'est sa propre définition de "système ». Cf. Michel de Certeau, « La rupture instauratrice, ou le christianisme dans la culture contemporaine », Esprit, nº 404, juin 1971, p. 1184, n. 13.

49. Il faut signaler qu'en 1996, la revue des dominicains anglais New Blackfriars (où de Certeau avait collaboré avec un texte en 1970) a essayé de réparer ce "manque » à travers un numéro spécial dédié à " Michel de Certeau SJ » sous la légende "The first collection of essays in the English langage devoted to Certeau's work from the perspective of a theologian ", New Blackfriars, Vol. LXXVII, n 909, november 1996, p. 477-528. La présence de Luce Giard, Joseph Moingt, Jeremy Ahearne, Ian Buchanan, Frederick Christian Bauerschmidt et Graham Ward, parmi d'autres collaborateurs, parle d'elle-même. Précisément, ce dernier, Professor of Contextual Theology and Ethics à l'Université de Manchester, a été l'un des principaux animateurs des quelques aspects de la pensée religieuse certalienne lesquels, pourtant, ne coïncident pas toujours avec le "premier » de Certeau. Voir à cet égard, les deux «Blackwell Readers" édités par lui : d'une part, The Postmodern God. A Theological Reader, lequel inclut deux essais de Michel de Certeau et une introduction rédigée par Frederick C. Bauerschmidt, Oxford, Blackwell, coll. « Blackwell Readings in Modern Theology ", 1997, p. 135-158 et d'autre part, une anthologie entièrement dédiée à Michel de Certeau, The Certeau Reader, Oxford, Blackwell, coll. «Blackwell Readers », 2000.

50. Pour une analyse plus précise des possibilités de mettre en rapport l'œuvre de Michel de Certeau avec les « cultural studies ", voir le travail de Ben Highmore, Michel de Certeau. Analysing Culture, London, Continuum, coll. « Arts \& Humanity ", 2006. La premisse de Highmore consiste à penser l'œuvre de Michel de Certeau comme «the study of inventive (and hopeful) methodologies in the face of epistemological doubt ", "Preface and Acknowledgements », p. xi.

51. C'est l'une des observations que Patrick Gudridge a faite sur l'ouvrage d'Ahearne: "Le rangement d'Ahearne a pourtant son prix. Pour la plupart, il aborde les écrits de Certeau tous ensemble: comme si eux-mêmes fussent un texte unique et illustratif d'un phénomène. Les questions qui pourraient être fragmentées deviennent marginales» [ "Ahearne's ordering, however, has its costs. For the most part, he treats Certeau's writings all together : as though one text, illustrative of one phenomenon. Questions that might be fragmenting become marginal »], in Patrick O Gudridge, "Michel de Certeau: Interpretation and Its Other by Jeremy Ahearne » (Reviewed Works), Contemporary Sociology, vol. XXVI, no 2, mars 1997, p. 251-252].

52. Je dois remercier Pierre Lardet qui m'a averti aimablement des risques d'un terme qui pourrait donner lieu à de forts malentendus. Dans le cadre général de notre recherche et dans la logique particulière de la production certalienne de "recueils ", on appelera "texte bref » l'écriture d'un texte sous forme d'«article» ou d'«essai» dont la porosité assure son appartenance à un ensemble plus vaste. À cet égard, chacun des écrits «brefs » de Michel de Certeau constitue un espace autonome et créatif garanti par une série de dispostifs qui fait partie des formes de sa cohérence interne : une temporalité narrative définie, une perspective explicite sur le sujet traité, un élan poétique manifeste et rhétorique assuré par de nombreuses figures à la manière d'une meraviglia, un propos politique sous-jacent exprimé à travers de différents degrés 
de lisibilité et une structure épistémologique rigoureuse déclarée par l'usage méthodique des sources.

53. «I have produced a thematic analysis, treating my corpus as though it were a syncronic collection, while also introducing diachronic nuances. This seems the most helpful way of introducing the reader in a limited space to both the breadth and rewarding complexity of Certeau's thought ", in Jeremy Ahearne, op. cit., «Introduction », p. 5.

54. À cette époque, les seules références biographiques consultables étaient, d'une part, celles écrites par Luce Giard (la première, une «Biobibliographie ", avait paru dans le volume collectif publié par le Centre Georges Pompidou: Michel de Certeau, Luce Giard, (dir.), p. 245-253 et la deuxième, c'est une « Notice » parue dans le dossier de Recherches de Science Religieuse, Luce Giard (dir.), Le voyage mystique. Michel de Certeau, p. 187-189) et, d'autre part, la petite et excellente introduction à la vie et à l'œuvre de Michel de Certeau écrite par Dominique Julia et Claude Rabant pour Universalia 1987 (Paris, 1987, p. 536-538).

55. Cf. Michel de Certeau. Les chemins d'histoire, Christian Delacroix, François Dosse, Patrick García, et Michel Trebitsch (dirs.), Paris, Complexe, coll. "Histoire du temps présent ", 2002; et le «Cahier» double, $\mathrm{n}^{\text {os }}$ 80/81 de la revue Espaces/Temps, "Michel de Certeau, histoire/ psychanalyse. Mises à l'épreuve", 2002. Ces deux publications sont en fait quelques unes des interventions d'une séminaire organisé par l'Institut d'Histoire du Temps Présent (IHTP) et par le Centre d'histoire culturelle des sociétés contemporaines de l'Université de Saint-Quentin-enYvelines entre 1998 et 2000. À ces deux travaux, il faut ajouter un autre écrit publié par François Dosse, Paul Ricour, Michel de Certeau. L'Histoire : entre le dire et le faire. Paris, L'Herne, coll. « Glose », 2006.

56. À la différence de l' « école française » (dont le projet envisage des objectifs à long terme liés aux enjeux des textes certaliens et à sa figure intellectuelle et religieuse), ainsi que du «mouvement anglophone» (dont l'énorme atomisation de lectures tend à faire de l'œuvre de Michel de Certeau un objet d'appropriation multiple à partir de toutes sortes de disciplines), le « courant français » (animé essentiellement par François Dosse à côté de Patrick Garcia, Christian Delacroix et Michel Trebitsch) s'inscrit dans une histoire intellectuelle du parcours certalien bien attachée à l'historiographie, ainsi qu'à l'anthropologie et à la psychanalyse par le biais de l'histoire. Tout d'abord, nous avons préféré le terme « courant » parce que sa durée correspond aux temps d'une conjoncture limitée aux résultats d'un séminaire collectif où la figure de Michel de Certeau est analysée en tant que «cas» de «la pertinence, dans l'écriture de l'histoire du temps présent, d'un certain nombre de notions et concepts utilisés par les historiens ", Christian Delacroix, et al. « Pourquoi Michel de Certeau aujourd'hui ?», op. cit., Michel de Certeau. Les chemins d'histoire, p. 15. À cet égard, la présence au sein de ce séminaire de Luce Giard, Jeremy Ahearne, Bruno Ribes, Mireille Cifali, Arlette Farge ou Roger Chartier, parmi bien d'autres, indique à quel point cet espace fut l'objet d'un échange « pluriel » et un lieu de circulation d'idées, un trait qui pourrait s'appliquer également aux travaux animés par l'« école française ». Il ne faut pas oublier non plus que tous les intellectuels qui ont participé à titre personnel à diverses discussions autour de l'œuvre de Michel de Certeau l'ont fait dans le cadre de ces deux "communautés interprétatives » et en dehors d'elles. C'est ainsi que de nombreuses revues lui ont consacré ou dédié en hommage (en France et à l'étranger) un numéro spécial. D'ailleurs, il est important de ne pas négliger qu'en dehors de la France et des pays anglophones, il existe plusieurs mouvements plus ou moins définis qui mettent l'accent sur différentes questions de l'œuvre certalienne, tels que le monde hispanophone (mexicain particulièrement) et lusophone (brésilien), italien et notamment allemand, tous renseignés par Luce Giard et Dominique Julia (art. cité, Esprit, nº 219, mars 1996, p. 132-135).

57. Il s'agit du fonds épistolier conservé aux archives de la Compagnie de Jésus de la province de France à Vanves. 
58. C'est en fait Jacques Revel qui, pour la première fois, mit en évidence ce rapport conflictuel (et paradoxal bien entendu) de Michel de Certeau aux institutions : «Sa longue pérégrination acadé-mique, de positions précaires en institutions lointaines, il l'a subie, bien sûr, et il en a parfois souffert; mais il l'a aussi, d'une certaine manière, revendiquée comme si elle rendait claire la logique d'une posture. À ses propres dépens, il s'est obstiné à frayer l'espace de la liberté dont il éprouvait le besoin ", Jacques Revel, «Michel de Certeau historien : l'institution et son contraire ", in Histoire, mystique et politique. Michel de Certeau, Luce Giard, Hervé Martin, et Jacques Revel, texte établi et présenté par Luce Giard et Pierre-Jean Labarrière, Paris, Jérôme Millon, (hors collection), 1991, p. 122. Pour ce qui touche cet interstice mentionné par Revel, il faudra se demander si effectivement ce rapport a fait de Michel de Certeau un marcheur « aux marges » de toute institution comme l'a signalé François Dosse.

59. La critique Martine Boyer-Weinmann classifie ce travail comme "biographie blanche», c'est-à-dire, celle qui «connaît ses degrés de blancheur et ne recoupe pas automatiquement l'opposition entre "biographie vulgarisée" et "biographie savante". La biographie blanche, c'est tout ce qui fait obstacle à la justification de l'orientation critique ». À l'égard du travail sur Michel de Certeau, elle affirme que Dosse «part du principe que chacun sait ce qu'est une biographie intellectuelle, et surtout pourquoi précisément ce biographe s'est intéressé à ce biographié. La non justification du choix méthodologique laisse le lecteur inférer (à tort sans doute) qu'un intellectuel (l'historien des idées François Dosse) n'avait pas d'autre ressource narrative à sa disposition que celle de la «biographie intellectuelle " pour dresser le portrait d'une grande figure intellectuelle, jésuite de surcroît. Ce mutisme sur le parti pris de l'enquête est d'autant plus regrettable que le même François Dosse avait réussi son pari, a priori plus difficile, de rédiger la biographie intellectuelle d'un sujet vivant et réticent, Paul Ricœur... ", Martine Boyer-Weinmann, La relation biographique. Enjeux contemporains, Seyssel, Éditions Champ Vallon, coll. « Essais », 2005, p. 111-112.

60. À ce sujet, Alfonso Mendiola, «Prefacio", in François Dosse (2002), Michel de Certeau. El caminante herido, traduit par Claudia Mascarua, Mexico, Universidad Iberoamericana, Departamento de Historia, coll. «El oficio de la Historia », 2003, p. 11-12.

61. Étienne Fouilloux (1998), Une église en quête de liberté. La pensée catholique française entre modernisme et Vatican II (1914-1962), Paris, Desclée de Brouwer, coll. « Anthropologiques », 2006. Voir aussi son essai d'« ego-histoire ", "Itinéraire d'une recherche », in Étienne Fouilloux, $A u$ cœur du XXe siècle religieux, Paris, Les Éditions Ouvrières, coll. «Églises/Sociétés », 1993, p. 7-21.

62. Étienne Fouilloux, op. cit., «Introduction », p. 14.

63. "The analyst of the process of re-employement was himself a great master of the art. Certeau had a remarkable gift for analogical thinking. As he became increasingly interested in secular problems, he approched them by making use of concepts that had already been employed both by himself and by others in the religious domain and adapting them to new purposes. His conceptual apparatus, or in the historian Lucien Febvre's famous phrase his outillage mental, was taken from religion and translated into secular terms", in Peter Burke, "The Art of ReInterpretation : Michel de Certeau ", Theoria. A Journal of Social and Political Theory, University of KwaZulu-Natal, Pietermaritzburg, no 100, décembre 2002, p. 27-37.

64. C'est le propre Ignace de Loyola qui dans son autobiographie et à partir d'un geste qui ne laisse d'évoquer la trace augustinienne, parle de lui comme du pèlerin. Cf. notamment l'édition consultée par de Certeau, Saint Ignace de Loyola, Le récit du pèlerin. Autobiographie de Saint Ignace de Loyola. Troisième édition entièrement refondue par A. Thiry, S.J. Bruges, Desclée de Brouwer, coll. «Museum Lessianum », section "Ascétique et Mystique " $\mathrm{n}^{\mathrm{o}}$ 15, 1956. Je dois remercier Pierre-Antoine Fabre des subtiles indications qu'il m'a données à propos des Jésuites français et de cet article dans son ensemble. 
65. Brian Stock (2008), Lire, une ascèse? Lecture ascétique et lecture esthétique dans la culture occidentale, Menahem Stern Jerusalem Lectures, 2005. Traduit de l'anglais par Christophe Carraud, Paris, Jérôme Millon, coll. « Nomina », 2008, ch. II « Le lecteur ascétique », p. 75-76.

66. Louis Beirnaert (1983), "Ignace de Loyola, fin ou transformation du pèlerinage ", in Louis Beirnaert, Aux frontières de l'acte analytique. La Bible, saint Ignace, Freud et Lacan, Paris, Seuil, [hors collection], 1987, p. 199-204.

67. Alphonse Dupront (1987), Du sacré. Croisades et pèlerinages. Images et langages, Paris, Gallimard, coll. « Bibliothèque des Histoires », 2005, § « Le fait pèlerin », p. 46.

68. Ibid., p. 48 .

69. Nous prenons la différence conceptuelle et fonctionnelle entre "savoir » et "discipline» d'après l'article de Gérard Lenclud, "L'anthropologie et sa discipline ", in Qu'est-ce qu'une discipline? Jean Boutier, Jean-Claude Passeron, et Jacques Revel (dir.), Paris, Éditions de l'École des Hautes Études en Sciences Sociales, coll. «Enquête », 2006, p. 69-93.

70. Quant au "nomadisme » contenu dans le concept certalien de «braconnage ", Éric Maigret a remarqué : "Le nomadisme renvoie pour sa part à cette aptitude qu'ont les individus de se nourrir de textes hétérogènes, contradictoires, et, réciproquement, à cette faculté qu'ont les textes de générer des communautés d'interprétations différentes ", Éric Maigret, "Les trois héritages de Michel de Certeau. Un projet éclaté d'analyse de la modernité ", Annales, vol. LV, nº 3, 2000, p. 511-549.

71. Daniel Frey, L'interprétation et la lecture chez Ricœur et Gadamer (Introduction), « Lire la lecture. Une introduction à l'herméneutique de la lecture », § « Naissance et histoire de la lecture », Paris, PUF, coll. «Études d'histoire et de philosophie religieuses », 2008, p. 5.

72. Comme l'a souligné François Hartog, «Cette localisation, précisément parce qu'elle n'assigne pas un lieu ni non plus l'autorise, mais qu'elle désigne une pratique, ne pouvait que ravir cet autre lecteur (lui aussi souvent interrogé sur son identité) qu'était Certeau ", François Hartog, «L'écriture du voyage ", in Luce Giard (edit.), Michel de Certeau, p. 126. Je remercie François Hartog des précieuses indications qu'il m'a données sur la «pensée mobile» de Michel de Certeau.

73. Michel de Certeau, "L'expérimentation d'une méthode : les Mazarinades de Christian Jouhaud », Annales ESC, tome XLI, mai-juin 1986, p. 507-512.

74. Christian Jouhaud, Mazarinades : la Fronde des mots, Paris, Aubier, coll. " Collection historique ", 1985.

75. Christian Jouhaud, "Vingt ans après ", in Mazarinades: la Fronde des mots (1985), Paris, Flammarion, coll. « Collection historique », 2009, p. I-XVII.

\section{AUTEUR}

ANDRÉS G. FREIJOMIL

EHESS/CRH 